\title{
Drug pipeline: 1Q13
}

\section{Laura DeFrancesco}

US Food and Drug Administration approvals continued at breakneck pace with 12 new molecular and biological entities. These included Roche's long-anticipated antibody-drug conjugate Kadcyla and Janssen's

Historic US regulatory approvals by drug class

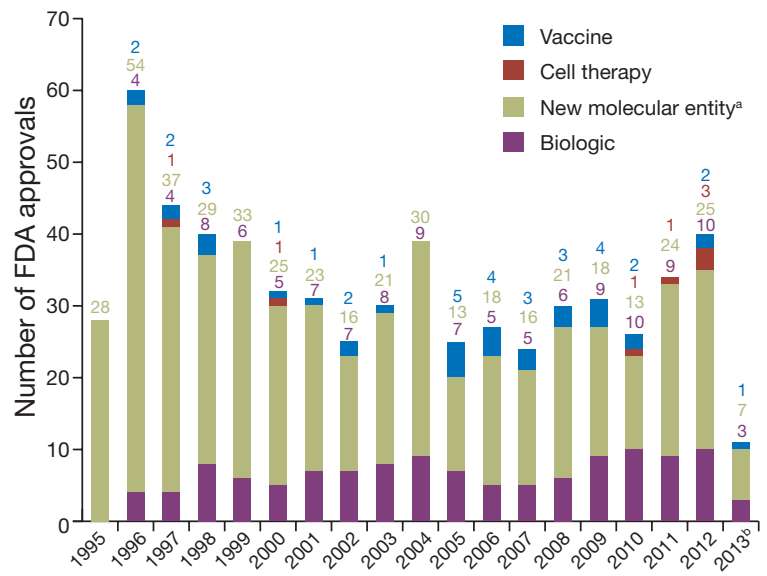

New molecular entity class includes mainly small-molecule drugs, but also steroid, synthetic peptide and mixe compounds. ${ }^{b}$ Partial year to March 29.

Source: U.S. Food and Drug Administration (FDA) (http://www.fda.gov), excluding non-NME drugs or new formulation

Notable regulatory setbacks (Q1 2013)

\begin{tabular}{|c|c|c|}
\hline Drug/company & Indication & Setback summary \\
\hline $\begin{array}{l}\text { Metreleptin } \\
\text { (r-metHuLeptin)/BMS }\end{array}$ & Lipodystrophy & $\begin{array}{l}\text { 1/31/13. Amylin announced plans to resubmit } \\
\text { BLA for this recombinant human leptin }\end{array}$ \\
\hline $\begin{array}{l}\text { Ampligen (rintatolimod)/ } \\
\text { Hemispherx }\end{array}$ & $\begin{array}{l}\text { Chronic fatigue } \\
\text { syndrome }\end{array}$ & $\begin{array}{l}\text { 2/4/13. FDA sent complete response letter for } \\
\text { polyl/polyC dsRNA immune activator, requesting } \\
\text { further studies on safety and efficacy }\end{array}$ \\
\hline $\begin{array}{l}\text { Ryzodeg (degludecplus)/ } \\
\text { Novo Nordisk }\end{array}$ & Diabetes & $\begin{array}{l}2 / 8 / 13 \text {. FDA sent a complete response letter } \\
\text { for this fixed combination of long-acting and } \\
\text { fast-acting insulins requesting additional cardio- } \\
\text { vascular data }\end{array}$ \\
\hline $\begin{array}{l}\text { Tresiba (insulin degludec)/ } \\
\text { Novo Nordisk }\end{array}$ & Diabetes & $\begin{array}{l}2 / 8 / 13 \text {. FDA sent a complete response letter for } \\
\text { this long-acting insulin requesting additional } \\
\text { cardiovascular data }\end{array}$ \\
\hline $\begin{array}{l}\text { Omontys (peginesatide)/ } \\
\text { Affymax }\end{array}$ & Kidney dialysis & $\begin{array}{l}2 / 22 / 13 \text {. FDA recall due to several cases of ana- } \\
\text { phylaxis, three leading to death }\end{array}$ \\
\hline Heplisav/Dynavax & $\begin{array}{l}\text { Hepatitis } \\
\text { vaccine }\end{array}$ & $\begin{array}{l}2 / 25 / 13 \text {. FDA sent complete response letter } \\
\text { requesting further evaluation of safety in broad } \\
\text { patient range ( } 18-70 \text { years) }\end{array}$ \\
\hline Kynamro/Sanofi Isis & $\begin{array}{l}\text { Dyslipidemia/ } \\
\text { hypercholester- } \\
\text { olemia }\end{array}$ & $\begin{array}{l}\text { 3/21/13. European CHMP panel confirmed } \\
\text { negative opinion after appeal by company }\end{array}$ \\
\hline CRLX101/Cerulean & $\begin{array}{l}\text { Non-small-cell } \\
\text { lung cancer }\end{array}$ & $\begin{array}{l}3 / 22 / 13 \text {. Company announced that phase } 2 \mathrm{~b} \\
\text { study of camptothecin-polymer conjugate failed } \\
\text { to meet endpoint of overall survival }\end{array}$ \\
\hline $\begin{array}{l}\text { Zymafos (palifosfamide)/ } \\
\text { Ziopharm Oncology }\end{array}$ & Sarcoma & $\begin{array}{l}\text { 3/26/13. Company suspended development of } \\
\text { bifunctional DNA alkylator after phase } 3 \text { trial } \\
\text { failed to meet primary endpoint of progression- } \\
\text { free survival }\end{array}$ \\
\hline Ixmyelocel-T/Aastrom & $\begin{array}{l}\text { Critical limb } \\
\text { ischemia }\end{array}$ & $\begin{array}{l}3 / 27 / 13 \text {. Company suspended phase } 3 \text { trial } \\
\text { because of recruitment problems. Phase } 2 \mathrm{~b} \text { trial } \\
\text { for dilated cardiac myopathy will continue. }\end{array}$ \\
\hline $\begin{array}{l}\text { Elacytarabine/Clavis } \\
\text { Pharma }\end{array}$ & AML & $\begin{array}{l}\text { 4/1/13. Company reported that phase } 3 \text { trial of } \\
\text { its lipid-conjugated form of cytarabine failed to } \\
\text { improve overall survival over control arm. }\end{array}$ \\
\hline
\end{tabular}

Source: BioMedTracker, a service of Sagient Research (http://www.biomedtracker.com/). FDA, US Food and Drug Administration; EMA, European Medicines Agency; BMS, Bristol-Myers Squibb; AML, acute myeloid leukemia; Administration; EMA, European Medicines Agency; BMS, Bristol-Mye first-in-class sodium glucose type 2 transporter Invokana. The quarter was not without disappointment, however; in particular, Affymax recalled its recently approved erythropoietin mimetic Omontys.

Notable upcoming regulatory decisions (Q3 2013)

\begin{tabular}{|c|c|c|}
\hline Drug/company & Indication & Expected regulatory decision \\
\hline Afatinib/Boehringer & $\begin{array}{l}\text { Non-small-cell } \\
\text { lung cancer }\end{array}$ & $\begin{array}{l}\text { 7/15/13, FDA PDUFA. Novel irreversible binder of EGFR } \\
\text { and HER2 }\end{array}$ \\
\hline Suvorexant/Merck & Insomnia & 5/20-5/21/13, FDA PDUFA. Orexin A and B antagonist \\
\hline $\begin{array}{l}\text { [18F]Flutemetamol/ } \\
\text { GE }\end{array}$ & $\begin{array}{l}\text { Alzheimer's } \\
\text { disease }\end{array}$ & $\begin{array}{l}\text { 6/25-7/25/13, FDA PDUFA. PET imaging agent for beta } \\
\text { amyloid plaques }\end{array}$ \\
\hline $\begin{array}{l}\text { Tivopath (tivozanib)/ } \\
\text { AVEO, Astellas }\end{array}$ & $\begin{array}{l}\text { Renal cell } \\
\text { carcinoma }\end{array}$ & $\begin{array}{l}7 / 26 / 13 \text {, FDA PDUFA. Small-molecule inhibitor of VEGF } \\
\text { receptors } 1,2,3\end{array}$ \\
\hline $\begin{array}{l}\text { Alpharadin (radium } \\
223 \text { chloride)/Bayer, } \\
\text { Algeta }\end{array}$ & Prostate cancer & $\begin{array}{l}8 / 14 / 13, \text { FDA PDUFA. Bone-targeting radium } 223 \\
\text { isotope }\end{array}$ \\
\hline $\begin{array}{l}\text { Florbetaben/Piramal } \\
\text { Health }\end{array}$ & $\begin{array}{l}\text { Alzheimer's } \\
\text { disease }\end{array}$ & $\begin{array}{l}\text { 9/3/13-10/3/13, PDUFA. F18-tagged sugar molecule } \\
\text { for detecting beta amyloid plaques }\end{array}$ \\
\hline
\end{tabular}

Notable clinical trial results (Q1 2013)

\begin{tabular}{|c|c|c|}
\hline Drug/company & Indication & Result summary \\
\hline $\begin{array}{l}\text { MEK162/ } \\
\text { Novartis }\end{array}$ & Melanoma & $\begin{array}{l}\text { 2/13/13. Phase } 2 \text { open-label, non-randomized clinical } \\
\text { trial showed efficacy on patients with NRAS-mutated or } \\
\text { Val600 BRAF mutations (Lancet Oncol. 14, 249-256) }\end{array}$ \\
\hline $\begin{array}{l}\text { Treanda } \\
\text { (bendamustine)/ } \\
\text { TEVA }\end{array}$ & $\begin{array}{l}\text { Mantle cell } \\
\text { lymphoma, } \\
\text { non-Hodgkin } \\
\text { lymphoma }\end{array}$ & $\begin{array}{l}\text { 2/20/13. In a randomized open-label phase } 3 \text { trial, this } \\
\text { novel DNA alkylating agent with Rituxan/MabThera (ritux- } \\
\text { imab) showed increased progression-free survival and } \\
\text { fewer side effects than the standard of care (Lancet } \mathbf{3 8 1} \text {, } \\
\text { 1203-1210) }\end{array}$ \\
\hline $\begin{array}{l}\text { Pradaxa } \\
\text { (dabigatran } \\
\text { etexilate)/ } \\
\text { Boehringer } \\
\text { Ingleheim }\end{array}$ & $\begin{array}{l}\text { Venous } \\
\text { thromboembolism }\end{array}$ & $\begin{array}{l}\text { 2/20/13. Two double-blind randomized phase } 3 \text { trials } \\
\text { show that this direct thrombin inhibitor reduces the risk } \\
\text { of recurrent embolisms ( } N \text {. Engl. J. Med. 368, 709-718) }\end{array}$ \\
\hline Enobosarm/Gtx & $\begin{array}{l}\text { Cachexia/weight } \\
\text { loss }\end{array}$ & $\begin{array}{l}\text { 3/14/13. In phase } 2 \mathrm{~b} \text { trial, patients showed significant } \\
\text { increase in body mass and stair climbing power. (Lancet } \\
\text { Oncol. 14, 335-345, 2013, doi:10.1016/S1470- } \\
\text { 2045(13)70055-X) }\end{array}$ \\
\hline
\end{tabular}

Source: BioMedTracker, a service a Sagient Research (http://www.biomedtracker.com/). FDA, US Food and Drug Administration; EMA, European Medicines Agency; NRAS, neuroblastoma rat sarcoma.

Notable regulatory approvals (Q1 2013)

\begin{tabular}{|c|c|c|}
\hline Drug/company & Indication & Drug information \\
\hline $\begin{array}{l}\text { Pomalyst (pomalidomide)/ } \\
\text { Celgene }\end{array}$ & Multiple myeloma & $\begin{array}{l}\text { 2/8/13, FDA. Cell signaling molecule via } \\
\text { cereblon (CBRN) with immunomodulatory } \\
\text { and anti-angiogenesis properties }\end{array}$ \\
\hline $\begin{array}{l}\text { Kadcyla (trastuzumab DM-1)/ } \\
\text { Roche }\end{array}$ & Breast cancer & $\begin{array}{l}\text { 2/22/13, FDA. Antibody-drug conjugate } \\
\text { against HER2 }\end{array}$ \\
\hline Stivarga (regorafenib)/Bayer & $\begin{array}{l}\text { Gastrointestinal } \\
\text { stromal tumors }\end{array}$ & $\begin{array}{l}2 / 25 / 13 \text {, FDA. Multi-kinase inhibitor } \\
\text { against various RAF kinases and VEGF } \\
\text { receptor family (already approved in colon } \\
\text { cancer) }\end{array}$ \\
\hline $\begin{array}{l}\text { TOBI (tobramycin) Podhaler/ } \\
\text { Novartis }\end{array}$ & Cystic fibrosis & $\begin{array}{l}\text { 3/22/13, FDA. First inhaled powder ami- } \\
\text { noglycoside antibiotic }\end{array}$ \\
\hline $\begin{array}{l}\text { Tecfidera (dimethyl fumarate)/ } \\
\text { Biogen }\end{array}$ & Multiple sclerosis & $\begin{array}{l}\text { 3/27/13, FDA. Oral neuroprotective } \\
\text { anti-inflammatory molecule targeting } \\
\text { NF-KappaB }\end{array}$ \\
\hline $\begin{array}{l}\text { Invokana (canaglifloxin)/ } \\
\text { Janssen (J\&J) }\end{array}$ & Type 2 diabetes & $\begin{array}{l}\text { 3/29/13, FDA. First-in-class sodium- } \\
\text { glucose co-transporter inhibitor }\end{array}$ \\
\hline
\end{tabular}

Source: BioMedTracker, a service of Sagient Research (http://www.biomedtracker.com/). FDA, US Food and Drug Administration; EMA, European Medicines Agency; J\&J, Johnson \& Johnson.

Laura DeFrancesco is Senior Editor at Nature Biotechnology. 